\title{
Impact of Sacubitril/Valsartan on Patient Outcomes in Heart Failure: Evidence to Date
}

This article was published in the following Dove Press journal:

Therapeutics and Clinical Risk Management

\section{Sara Akbar \\ Nitin Kabra \\ Wilbert S Aronow \\ 'Department of Cardiology, Westchester Medical Center, Valhalla, NY 10595, USA; ${ }^{2}$ Department of Medicine, New York Medical College, Valhalla, NY 10595, USA}

Correspondence: Wilbert S Aronow Director of Cardiology Research, Department of Cardiology, Westchester Medical Center, Macy Pavilion, Room I4I, Valhalla, NY 10595, USA

$\mathrm{Tel}+$ I (9|4)-493-53 I I

Fax +I (9|4)-235-6274

Email wsaronow@aol.com
Abstract: With an estimated 6.2 million adults affected in the USA, heart failure remains a leading cause of morbidity, mortality, and health-care costs, despite the use of guidelinebased medical therapies. The search for a more efficient therapy was rekindled when findings from the Prospective Comparison of Angiotensin Receptor-Neprilysin Inhibitor With Angiotensin-Converting Enzyme Inhibitor to Determine Impact on Global Mortality and Morbidity in Heart Failure (PARADIGM-HF) trial demonstrated evidence for cardiovascular and mortality benefit of sacubitril/valsartan, a dual angiotensin receptor blocker and neprilysin inhibitor (ARNI), over enalapril (an angiotensin-converting enzyme inhibitor) in patients with heart failure and reduced rjection fraction (HFrEF). Following the trial's compelling results, recommendations for the use of sacubitril/valsartan as a replacement for an angiotensin-converting enzyme inhibitor and/or angiotensin receptor blocker were incorporated into the 2016 American College of Cardiology (ACC), the American Heart Association (AHA), and the Heart Failure Society of America recommended (HFSA) guidelines for the management of heart failure. This review aims to gain insight into the benefits as well as limitations associated with the use of sacubitril/valsartan in the treatment of heart failure (HF) through exploration of various subgroup analyses of the PARADIGM-HF trial, subsequent retrospective analyses, and randomized controlled trials that followed this landmark trial.

Keywords: heart failure with reduced ejection fraction, angiotensin receptor-neprilysin inhibitor, sacubitril/valsartan, heart failure hospitalization, cardiovascular death, clinical outcome

\section{Introduction}

Heart failure (HF) is a clinical syndrome characterized by dyspnea, easy fatigability, and fluid retention. It stems from an imbalance in the homeostasis between structural, functional, and neurohumoral factors which result in the impairment of ventricular filling or ejection of blood after a pathological insult.

The initial response to any pathological insult to the cardiac tissue is the activation of the vasoconstrictor and anti-natriuretic systems (the sympathetic nervous system [SNS], the renin-angiotensin-aldosterone system [RAS], the arginine vasopressin system, and endothelin) which leads to increasing afterload on an already damaged heart as a result of increased sympathetic tone and cardiac remodeling and hypertrophy as a result of fluid retention and fibrotic changes in the myocardium. ${ }^{1-3}$ This is physiologically counterbalanced by vasodilator and natriuretic systems (the prostaglandin system [PGS], the nitric oxide system [NO], the dopaminergic system, and the natriuretic peptide system [NPS]). ${ }^{1-3}$ 
The complex pathophysiological interactions between the above-noted systems have recently therefore been the mainstay of development of targeted medical therapy. The conventional therapies for heart failure with reduced ejection fraction (HFrEF) include beta-blockers targeting the SNS, while angiotensin-converting enzyme inhibitors (ACEI), angiotensin receptor blockers (ARBs), and mineralocorticoid receptor antagonist (MRA) target the overactivated RAS on pathologic ventricular remodeling. ${ }^{4}$

However, despite advancement in the HF pharmacotherapy, approximately 6.2 million adults were diagnosed with heart failure between 2013 and 2016 (compared to 5.7 million adults from 2009 to 2012 recorded by the National Health and Nutrition Examination Survey) in the USA. ${ }^{5}$ HF exacerbations in the USA result in an estimated one million hospitalizations yearly and contributes largely to the overall HF health-care expenditure. ${ }^{5}$ Epidemiological data further indicate that from the patients hospitalized with heart failure between 2005 and 2010, half had HFrEF and half had heart failure with preserved ejection fraction (HFpEF). ${ }^{6}$ Heart failure is more prevalent in individuals greater than 65 years of age, ${ }^{7}$ in African Americans ${ }^{8}$, and in men compared to women. ${ }^{5}$ The increased prevalence within these groups may be attributed to a higher incidence of cardiovascular risk factors, including hypertension, obesity, and diabetes mellitus. ${ }^{9}$

A prediction holds that the prevalence of heart failure will increase by $46 \%$ from 2012 to $2030 .^{5}$ This prediction combined with the rising overall cost of HF (approximately $\$ 30.7$ billion in 2012$)^{10}$ necessitates the development of better therapies that aim to reduce hospitalization and morbidity associated with HF. Therefore, newer therapies that have emerged in the past decade have shifted focus to the counter-regulatory pathways described earlier. In particular, natriuretic peptides and counteracting degradation of these peptides via inhibition of neprilysin or neutral endopeptidase have gained considerable interest.

The landmark PARADIGM-HF (Prospective Comparison of Angiotensin Receptor-Neprilysin Inhibitor With Angiotensin-Converting Enzyme Inhibitor to Determine Impact on Global Mortality and Morbidity in Heart Failure) trial demonstrated that sacubitril/valsartan, a dual angiotensin receptor blocker and neprilysin inhibitor (ARNI), is superior to enalapril, ${ }^{11}$ a drug known for its morbidity and mortality benefits in patients with HFrEF. ${ }^{12}$ Since the completion of this trial in 2013, sacubitril/valsartan has been an important area of study, and this review aims to assess the impact of this drug on patient outcomes by investigating current and ongoing randomized controlled trials as well as retrospective analyses.

\section{Development of Combination Therapy}

As discussed earlier, the past decade witnessed increased interest in exploring the NPS to counter the remodeling effects of pharmacotherapy targeting the SNS and RAS. However, initial studies using solely natriuretic peptides such as intravenous nesiritide (recombinant human BNP) did not show sustained benefits in HF mortality or readmissions ${ }^{13,16}$ nor was there any improvement in clinical status or quality of life scores seen in a study using candoxatril, a neprilysin inhibitor (NEPI), as sole therapy. ${ }^{17}$ These studies postulated that in addition to degrading natriuretic peptides, neprilysin also degrades vasodilators and vasoconstrictors. ${ }^{17}$ Therefore, the neprilysin inhibitors also potentiate vasoconstrictors like angiotensin II as well as vasodilators, thereby neutralizing any beneficial effects. ${ }^{18}$

To resolve this dilemma, the OVERTURE (Omapatrilat Versus Enalapril Randomized Trial of Utility in Reducing Events) trial comparing combination omapatrilat (NEPI)enalapril (ACEI) with enalapril showed a modest reduction in heart failure hospitalizations in chronic HF patients with the combination drug; however, the greater frequency of angioedema compared to enalapril alone limited the benefits of the NEPI-ACEI. ${ }^{19}$

\section{Sacubitril/Valsartan: Mechanism of Action}

Sacubitril/valsartan, the first ARNI in 1:1 combination of sacubitril and valsartan, was developed to benefit from the combination of NEPI with ARB while also minimizing the risk of angioedema that limited the NEPI-ACEI combination. $^{10,20}$

Sacubitril (AHU377) is a prodrug that inhibits neprilysin through the active metabolite LBQ657, leading to increased levels of endogenous vasoactive peptides such as atrial natriuretic peptide (ANP), brain natriuretic peptide (BNP), c-type natriuretic peptide (CNP), bradykinin, and adrenomedullin. ${ }^{6}$

Valsartan, an ARB, displaces angiotensin II from the angiotensin 1 receptor; and antagonizes angiotensin 1-induced vasoconstriction as well as aldosterone release, catecholamine release, and arginine vasopressin release. ${ }^{21}$

Combination of these two drugs sparked an interest in HF pharmacotherapy that to date continues to be explored. 


\section{PARADIGM-HF Trial}

The PARADIGM-HF trial was a Phase III multi-center double-blind controlled trial that randomized 8442 patients of at least 18 years of age with New York Heart Association (NYHA) class II, III, or IV symptoms, an ejection fraction of $35 \%$ or less, and BNP of at least 150 $\mathrm{pg} / \mathrm{mL}$ (or $\geq 100 \mathrm{pg} / \mathrm{mL}$ if hospitalized for HF in previous 12 months) or elevated N-terminal pro-BNP (NT-proBNP) level $\geq 600 \mathrm{pg} / \mathrm{mL}$ (or $\geq 400 \mathrm{pg} / \mathrm{mL}$ if hospitalized for HF in previous 12 months) into either the sacubitril/valsartan group or the enalapril group. ${ }^{11}$

Sacubitril/valsartan was approved by the Food and Drug Administration (FDA) in 2015 after the results of the PARADIGM-HF trial showed a reduction in the combined primary outcome of death from cardiovascular causes and hospitalization in patients with HFrEF (ejection fraction $35 \%$ or less, and NYHA class II-IV) compared with enalapril.

A total of $558(13.3 \%)$ subjects in the sacubitril/valsar$\tan$ group and $693(16.5 \%)$ subjects in the enalapril group died from cardiovascular causes (hazard ratio, $0.80 ; 95 \%$ confidence interval [CI], 0.71 to $0.89 ; P<0.001) .{ }^{11}$ The majority of these cardiovascular deaths were from sudden cardiac death and deaths from worsening $\mathrm{HF}^{22}$

Sacubitril/valsartan also reduced the risk of hospitalization by $21 \%(P<0.001)$, and the patients were less likely to require intensive care admissions (768 versus $879 ; 18 \%$ rate reduction, $P=0.005$ ), intravenous positive inotropic agents ( $31 \%$ risk reduction, $P<0.001$ ), implantation of a heart failure device or cardiac transplantation (22\% risk reduction, $P=0.07$ ) and were $29 \%$ less likely to be rehospitalized for HF compared with those treated with enalapril $(p=0.001){ }^{23}$

There was an improvement in symptoms as measured by the Kansas City Cardiomyopathy Questionnaire (KCCQ) at 8 months (between-group difference, 1.64 points; $95 \% \mathrm{CI}$, 0.63 to $2.65 ; p=0.001$ ) in patients receiving sacubitril/valsartan as compared to enalapril. ${ }^{11}$ Compared to the enalapril group, however, the sacubitril/valsartan group had higher proportions of patients with hypotension (14\% versus $9.2 \%$, $p<0.001)$ and non-serious angioedema (19 versus 10$)$ but lower incidence of renal impairment (denoted by creatinine $\geq 2.5 \mathrm{mg} / \mathrm{dL} ; 3.3 \%$ versus $4.5 \% p=0.007$ ), hyperkalemia ( $4.3 \%$ versus $5.6 \%, p=0.007)$, and cough $(11.3 \%$ versus $14.3 \%, p<0.001) .{ }^{11}$ Drug discontinuation due to adverse events was observed in $10.7 \%$ of the sacubitril/valsartan group versus $2.3 \%$ of the enalapril group $(p=0.03) .{ }^{11}$
Additionally, there was no significant difference in the number of patients who discontinued therapy due to hypotension between the two groups $(0.9 \%$ in the sacubitril/valsartan group versus $0.7 \%$ in the enalapril group). ${ }^{11}$

These impressive results also led to the American College of Cardiology (ACC), the American Heart Association (AHA), and the Heart Failure Society of America (HFSA) updating their guidelines for the management of heart failure in 2016 to include ARNI therapy for patients with NYHA stage II-III HFrEF as a replacement of an angiotensin-converting enzyme inhibitor or angiotensin receptor blocker. ${ }^{24}$ The guideline additionally stated that an ARNI should not be used within 36 hours of use of an angiotensin-converting enzyme inhibitor nor should it be prescribed to patients with a history of angioedema. ${ }^{24}$

The PARADIGM-HF trial created immense interest in sacubitril/valsartan as it stimulated research into an otherwise relatively stagnant HF pharmacotherapy and encouraged further research into questions unanswered by the study.

\section{The PARADIGM-HF Trial and Real-World Eligibility}

Despite the impressive results of the trial, concerns were raised regarding the underrepresentation of certain groups in the study population such as NYHA class IV patients, black subjects, female subjects, and relatively older subjects (since median patient age in the trial was 63.8 years, which is lower than the real-world HFrEF population). ${ }^{7,11}$ It also excluded chronic kidney disease (CKD) stage 4 and 5 (eGFR $<30 \mathrm{~mL} / \mathrm{min} / 1.73 \mathrm{~m}^{2}$ ) patients, patients who had an acute coronary event within the last 3 months of randomization, and patients recently hospitalized for HF exacerbation (since the inclusion criteria required for patients to be taking stable doses of a $\beta$-blocker and an angiotensin-converting enzyme inhibitor or angiotensin receptor blocker for at least 4 weeks prior to randomization). ${ }^{11,25}$

One post hoc analysis of the PARADIGM-HF trial indicated that $60 \%$ of the study population had ischemic cardiomyopathy (ICM), which raises concern of the benefit of sacubitril/valsartan in patients with non-ischemic cardiomyopathy (NICM) ${ }^{26}$ However, the authors concluded that despite demographic differences, the primary composite endpoint of reduced cardiovascular death with sacubitril/valsartan over enalapril was consistent across the different etiology subgroups. ${ }^{26}$

Another post hoc analysis of the PARADIGM-HF trial demonstrated a consistent clinical benefit of sacubitril/ 
valsartan across geographic regions, despite having baseline regional demographic variations. ${ }^{27}$ These findings are encouraging for the use of sacubitril/valsartan in $\mathrm{HFrEF}$ patients to provide a reduction in HF morbidity and mortality across the world. Therefore, despite underrepresentation of certain subgroups in the PARADIGM-HF trial that seemingly limits sacubitril/valsartan's real-world eligibility, the drug's favorable outcomes in patients with HFrEF encouraged subsequent trials to investigate those subgroups and expand its current indications.

\section{Effects on Cardiac Remodeling and Aortic Stiffness}

The PROVE-HF trial in 2018 investigated the effects of sacubitril/valsartan therapy on biomarkers, myocardial remodeling, and outcomes. This trial monitored NTproBNP levels and changes in cardiac remodeling based on various echocardiographic parameters including LVEF, LV end-diastolic volume index (LVEDVI), LV end-systolic volume index (LVESVI), left atrial volume index (LAVI), and the ratio of early transmitral Doppler velocity/early diastolic annular velocity $\left(\mathrm{E} / \mathrm{e}^{\prime}\right)(p<0.001$ for all $) .{ }^{28}$ The results of this study showed that the baseline median NTproBNP concentration of $816 \mathrm{pg} / \mathrm{mL}$ was decreased to 455 $\mathrm{pg} / \mathrm{mL}$ at 12 months (difference, $p<0.001$ ) ${ }^{28}$ For the same period, the mean LVEF increased from $28.2 \%$ to $37.8 \%$; LVEDVI decreased from 86.93 to $74.15 \mathrm{~mL} / \mathrm{m}^{2}$; LVESVI decreased from 61.68 to $45.46 \mathrm{~mL} / \mathrm{m}^{2}$; and LVESVI decreased from 61.68 to $45.46 \mathrm{~mL} / \mathrm{m}^{2} \quad(p<0.001$ for all). ${ }^{28}$ The fact that the majority of these changes were seen early in the trial, including changes in NT-proBNP at lower doses of sacubitril/valsartan, has important consequences for starting sacubitril/valsartan early in the disease course.

The EVALUATE-HF trial (Study of Effects of Sacubitril/Valsartan versus Enalapril on Aortic Stiffness in Patients With Mild to Moderate HF With Reduced Ejection Fraction) in 2019 did not show any significant reduction in central aortic stiffness in patients with HFrEF when given sacubitril/valsartan as compared to enalapril. ${ }^{29}$ However, similar to the PROVE-HF trial, the study findings of greater reduction in left atrial volume, LVEDI, and LVESVI in patients treated with sacubitril/valsartan as compared to enalapril provide further insight into remodeling mechanisms underlying the beneficial effects of sacubitril/valsartan in HFrEF. ${ }^{29}$

\section{Initiation and Titration of Sacubitril/ Valsartan}

The optimal timing for initiation or up-titration of ARNI has been a focus of interest considering the drug's important benefits on CV morbidity, mortality, and hospitalization.

The TITRATION trial in 2018 studied the tolerability of initiating and up-titrating sacubitril/valsartan from $50 \mathrm{mg}$ to $200 \mathrm{mg}$ twice daily (the dose used in PARADIGM-HF trial) in patients with HFrEF. Patients were randomly allocated to two arms: 'condensed' where patients took sacubitril/valsar$\tan 100 \mathrm{mg}$ twice daily for 2 weeks followed by $200 \mathrm{mg}$ twice daily, whereas in the 'conservative' arm the patients took sacubitril/valsartan $50 \mathrm{mg}$ twice daily for 2 weeks, $100 \mathrm{mg}$ twice daily for 3 weeks, followed by $200 \mathrm{mg}$ twice daily. ${ }^{30}$ The results indicated that gradual up-titration optimized achievement of the target dose over 3 or 6 weeks. ${ }^{30}$ Additionally, pre-defined tolerability criteria of hypotension, renal dysfunction, hyperkalemia, and angioedema were seen more in 'condensed' versus 'conservative' arms $(9.7 \%$ versus $8.4 \%(p=0.570), 7.3 \%$ versus $7.6 \% \quad(p=0.990), 7.7 \%$ versus $4.4 \%(p=0.114)$, and $0.0 \%$ versus $0.8 \%$ of patients, respectively). ${ }^{30}$

In August 2019, the TRANSITION-HF trial was done to assess the tolerability and optimal timing for the initiation of sacubitril/valsartan after stabilization of patients with ADHF either $\geq 12$-h pre discharge or at any point post discharge (up to 14 days). ${ }^{31}$ The starting dose was either $24 / 26 \mathrm{mg}$ or 49 / $51 \mathrm{mg}$ twice daily with titration based on tolerability. ${ }^{31}$ The primary endpoint was the proportion of patients achieving a twice daily $97 / 103 \mathrm{mg}$ target dose after 10 weeks. $^{31}$ Comparable proportions of patients in the pre- and postdischarge initiation groups met the primary endpoint (45.4\% versus $50.7 \%$; risk ratio [RR] 0.90 ; $95 \%$ CI 0.79 to 1.02). ${ }^{31}$ A subgroup analysis of the TRANSITION study showed that as compared to patients with prior HFrEF, the newly diagnosed HFrEF patients achieved target dose at Week 10 (56\% versus 45\%; relative risk ratio $1.30,95 \%$ CI 1.12 to $1.52, P<0.001$ ), and fewer had serious adverse effects and permanent treatment discontinuations. ${ }^{32}$ These patients also showed greater decreases in N-terminal pro-BNP, lower rates of $\mathrm{HF}$ and all-cause re-hospitalisation as compared to patients with previous history of $\mathrm{HFrEF}^{32}$

In February 2019, the PIONEER-HF trial (The Comparison of Sacubitril/Valsartan versus Enalapril on Effect on NT-proBNP in Patients Stabilized from an Acute Heart Failure Episode) provided evidence of a reduction in the N-terminal pro-B-type natriuretic peptide (NT-proBNP) 
concentration with ARNI therapy compared to enalapril therapy (percent change, $-46.7 \%$ versus $25.3 \%$; ratio of change with sacubitril/valsartan versus enalapril, $0.71 ; 95 \%$ CI, 0.63 to $0.81 ; P<0.001)$ as early as one week into therapy. ${ }^{33}$ The study participants included patient populations underrepresented in PARADIGM-HF; including patients admitted for new HF, acute decompensated HF, patients not on stable conventional medications for $\mathrm{HF}$ including RAS inhibitors, and African-Americans. ${ }^{33,34}$

In November 2019, Martens et al conducted a retrospective analysis of heart failure patients with HFrEF who received sacubitril/valsartan for a class-IB indication in a tertiary heart failure clinic to ascertain determinants of maximal dose titration. ${ }^{35}$ Results indicated that younger age ( $\mathrm{HR}=0.862 ; \mathrm{CI}=0.751$ to 0.989$)$, higher systolic blood pressure $(\mathrm{HR}=1.077 ; \mathrm{CI}=1.014$ to 1.137$)$, lower serum creatinine ( $\mathrm{HR}=0.064 ; \mathrm{CI}=0.005$ to 0.822$)$, and higher previous dose of renin-angiotensin system-inhibitors (RASi $[\mathrm{HR}=1.065$; $\mathrm{CI}=1.016$ to 1.115$]$ ) independently predicted a higher odds of tolerating a maximal dose of sacubitril/valsartan. ${ }^{35}$

These studies highlight the importance of early intervention with gradual up-titration of sacubitril/valsartan in delaying disease progression in patients with HFrEF.

\section{Sacubitril/Valsartan and CKD}

The lower incidence of renal impairment and hyperkalemia noted in the sacubitril/valsartan group compared to the enalapril group in the PARADIGM-HF trial raised questions regarding possible nephroprotective effects of the drug.

In order to evaluate these nephroprotective benefits of sacubitril/valsartan the UK HARP-III trial (United Kingdom Heart and Renal Protection-III), a randomized double-blind trial, was conducted in 2017-2018. The results showed that over 12 months, although there was no difference in measured GFR: 29.8 (SE 0.5) among those assigned sacubitril/ valsartan versus 29.9 (SE 0.5$) \mathrm{mL} / \mathrm{min} / 1.73 \mathrm{~m}^{2}$ compared to those assigned irbesartan; however, sacubitril/valsartan lowered blood pressure and cardiac biomarkers in people with chronic kidney disease (CKD). ${ }^{36}$ The trial also provided evidence for safe use of sacubitril/valsartan in patients with CKD.

\section{Sacubitril/Valsartan and Diabetes Mellitus}

One observation of the PARADIGM-HF trial interestingly showed that patients with diabetes mellitus and pre-diabetes had a higher risk of the primary endpoint. ${ }^{11} \mathrm{~A}$ post hoc analysis of the PARADIGM-HF trial found that sacubitril/ valsartan decreased hemoglobin A1C levels by $0.26 \%$ during the first year of follow-up, compared to a reduction of $0.16 \%$ with enalapril $(p=0.0023) .{ }^{37}$ In addition, compared to enalapril, $29 \%$ fewer patients treated with sacubitril/valsartan initiated insulin therapy to achieve glycemic control $(7 \%$ versus $10 \%$ patients, hazard ratio $0.71,95 \% \mathrm{CI}: 0.56$ to $0.90 ; p=0.0052) .^{37}$ These results hypothesize an additional benefit of sacubitril/valsartan in HFrEF patients, many of whom may have diabetes mellitus as one of their comorbidities as discussed earlier.

\section{Sacubitril/Valsartan in HFpEF}

The PARAMOUNT-HF (Prospective Comparison of ARNI with ARB on Management of Heart Failure with Preserved Ejection Fraction) trial in 2012 was the earliest trial of ARB and ARNI which showed that in patients with HFpEF, sacubitril/valsartan reduced NT-proBNP to a greater extent than did valsartan at 12 weeks (ratio sacubitril/valsartan/valsartan, $0.77,95 \%$ CI 0.64 to 0.92 , $p=0.005){ }^{38}$ At 36 weeks, improvements in left atrial size and NYHA functional class demonstrated the beneficial effects on myocardial damage and left atrial remodeling. ${ }^{38}$

In 2019, the Prospective Comparison of ARNI With ARB Global Outcomes in HF With Preserved Ejection Fraction (PARAGON-HF) trial studied the effect of ARNI on patients with HFpEF. The results of the study showed that in patients with HFpEF (ejection fraction $45 \%$ or more), as compared to valsartan, ARNI did not significantly lower the rate of HF hospitalizations and cardiovascular deaths. ${ }^{39}$ A subgroup analysis suggested a possible benefit of ARNI in patients with a lower left ventricular ejection fraction (LVEF approximately 45 to $57 \%$ ) in whom ARNI may have the same biologic effect as it does in those with HFrEF. ${ }^{40}$ This analysis as well as another noted that sacubitril/valsartan seemed to reduce the risk of heart failure hospitalization more in women than in men as compared to valsartan. ${ }^{40,41}$

The PARALLAX trial is a currently ongoing randomized double-blind controlled study comparing LCZ696 to medical therapy for comorbidities in HFpEF patients that aims to assess for superiority of sacubitril/valsartan (LCZ696) over established guideline-directed medical therapies including enalapril and valsartan in reducing NT-proBNP as well as in improving exercise capacity and HF symptoms. ${ }^{42}$

\section{Further Investigation}

Several trials are currently ongoing to clarify as well as explore benefits of sacubitril/valsartan in HF management. 
In this review we will discuss some of these trials to gain perspective into the future of sacubitril/valsartan therapy.

The Study of Efficacy and Safety of LCZ696 in Japanese Patients With Chronic Heart Failure and Reduced Ejection Fraction (PARALLEL-HF) is a Phase III clinical trial that aims to assess whether the reduction in cardiovascular deaths and HF hospitalizations with use of sacubitril/valsartan as compared to enalapril seen in the PARADISE-HF trial can be replicated in the Japanese population ${ }^{43}$ and therefore assess the generalizability of the trial results in a real-world HF population.

The Prospective ARNI versus ACE Inhibitor Trial to Determine Superiority in Reducing Heart Failure Events After Myocardial Infarction (PARADISE-MI) trial is currently ongoing to evaluate the effect of inpatient sacubitril/ valsartan versus ramipril in reducing cardiovascular death and HF hospitalization in post-acute myocardial infarction patients with evidence of LV systolic dysfunction $(\mathrm{EF}<40 \%)$ and/or pulmonary congestion, with no known prior history of chronic $\mathrm{HF}^{44}$

The Effects of Sacubitril/Valsartan Compared to Valsartan on Left Ventricular Remodelling in Asymptomatic Left Ventricular Systolic Dysfunction After Myocardial Infarction (RECOVER-LV) trial, unlike the PARADISE-MI trial, compares sacubitril/valsartan with valsartan and aims to provide a mechanistic explanation for cardiovascular benefits in patients with LV systolic dysfunction after myocardial infarction using both cardiac imaging and cardiac biomarkers. ${ }^{45}$

Differential Vascular and Endocrine Effects of Valsartan/ Sacubitril in Heart Failure With Reduced Ejection Fraction (VASCEND) is a double-blind randomized controlled Phase IV trial that aims to evaluate whether sacubitril/valsartan leads to a greater improvement in endothelial function (considering neprilysin inhibition increases vasoactive peptides) and endocrine imbalance compared to valsartan alone. ${ }^{46}$

The Safety and Efficacy of Angiotensin Receptorneprilysin Inhibitor After Left Ventricular Assist Device Implant (SEAL-IT) trial aims to evaluate how well tolerated and effective an angiotensin receptor-neprilysin inhibitor (sacubitril/valsartan) is in patients with contemporary durable continuous flow left ventricular assist device (CFLVAD) implantation compared to usual-care oral vasodilator therapy. ${ }^{47}$

The Evaluation in Real Life Conditions of Sacubitril/ valsartan Combination in Patients With Chronic Heart Failure and Sleep Apnea Syndrome (the SACUBITRIL/ VALSARTAN-SAS) study aims to evaluate whether using sacubitril/valsartan in HF patients presenting with sleep apnea syndrome (SAS) could improve SAS and in particular the central component of the apnea-hypopnea index. ${ }^{48}$

\section{Limitations of Sacubitril/Valsartan}

As discussed earlier, in the PARADIGM-HF trial, sacubitril/ valsartan was associated with higher proportions of patients with hypotension and non-serious angioedema compared to enalapril. ${ }^{11}$ In both the PARADIGM-HF trial and PARAMOUNT trials, symptomatic hypotension was the most common adverse event reported with sacubitril/valsar$\tan$ with a frequency of $18 \%$ and $19 \%$, respectively. ${ }^{11,38}$ The risk of other adverse effects of ARNI use such as hyperkalemia, cough, and worsening renal function have been shown to be lower than ACEI use alone. ${ }^{11}$

In 2018, Perlman et al analyzed the FDA adverse event report system database to evaluate cognition and dementia related adverse effects (CDRAE) with sacubitril/ valsartan. ${ }^{49}$ Since neprilysin is involved in the degradation of amyloid-beta, there is concern that sacubitril/valsartan could increase the risk for dementia. ${ }^{49}$ However, based on the results of the study sacubitril/valsartan was associated with CDRAE in 459 reports $(5.1 \%)$, but this was lower than the proportion of these reports among other medications $(6.6 \%$, reporting odds ratio [ROR] $0.72,95 \%$ CI 0.65 to 0.79$).{ }^{49}$ More studies need to be conducted in order to evaluate for long term cognitive effects that the drug might have.

An additional adverse effect of sacubitril/valsartan includes teratogenicity from its ARB component which precludes that this medication should be avoided in pregnancy.

\section{Conclusion}

These ongoing clinical trials (as well as many other others not covered in this review) and their subsequent post hoc analyses will ensure continual expansion of our understanding of patient outcomes of sacubitril/valsartan in treatment of HF.

\section{Funding}

This research received no specific grant from any funding agency in the public, commercial, or not-for-profit sectors.

\section{Disclosure}

The authors declare that there is no conflict of interest. 


\section{References}

1. Polónia J, Gonçalves FR. The historical evolution of knowledge of the involvement of neurohormonal systems in the pathophysiology and treatment of heart failure. Revista Portuguesa De Cardiologia. English Edition. $\quad<$ italic $>$ Rev Port Cardiol</italic $>$. 2019; 38;883-895.

2. Anastasios L, Giuseppe R, Koch WJ. Adrenergic nervous system in heart failure. Circ Res. 2013;113(6):739-753. doi:10.1161/ CIRCRESAHA. 113.300308

3. Iwanaga Y, Nishi I, Furuichi S, et al. B-type natriuretic peptide strongly reflects diastolic wall stress in patients with chronic heart failure: comparison between systolic and diastolic heart failure. $J \mathrm{Am}$ Coll Cardiol. 2006;47(4):742-748. doi:10.1016/j.jacc.2005.11.030

4. Reed BN, Street SE, Jensen BC. Time and technology will tell: the pathophysiologic basis of neurohormonal modulation in heart failure. Heart Fail Clin. 2014;10(4):543-557. doi:10.1016/j.hfc.2014.07.002

5. Benjamin EJ, Muntner P, Bittencourt MS. Heart disease and stroke statistics-2019 update: a report from the American Heart Association. Circulation. 2019;139(10):e5628.

6. Steinberg BA, Zhao X, Heidenreich PA, et al. Trends in patients hospitalized with heart failure and preserved left ventricular ejection fraction: prevalence, therapies, and outcomes. Circulation. 2012;126 (1):65-75. doi:10.1161/CIRCULATIONAHA.111.080770

7. Huffman MD, Berry JD, Ning H, et al. Lifetime risk for heart failure among white and black Americans: cardiovascular lifetime risk pooling project. J Am Coll Cardiol. 2013;61(14):1510-1517. doi:10.1016/ j.jacc.2013.01.022

8. Bahrami H, Ronal R, Bluemke DA, et al. Differences in the incidence of congestive heart failure by ethnicity: the multi-ethnic study of atherosclerosis. Arch Intern Med. 2008;168(19):2138-2145. doi:10.1001/archinte.168.19.2138

9. Bergsten TM, Nicholson A, Donnino R, et al. Predicting adults likely to develop heart failure using readily available clinical information: an analysis of heart failure incidence using the NHEFS. Prev Med. 2020;Jan(130):105878. doi:10.1016/j.ypmed.2019.105878

10. McMurray JJ, Petrie MC, Murdoch DR, Davie AP. Clinical epidemiology of heart failure: public and private health burden. Eur Heart J. 1998;19:P916.

11. McMurray JJ, Packer M, Desai AS, et al. Angiotensin-neprilysin inhibition versus enalapril in heart failure. $N$ Engl J Med. 2014;11 (371):993-1004. doi:10.1056/NEJMoa1409077

12. SOLVD Investigators*. Effect of enalapril on survival in patients with reduced left ventricular ejection fractions and congestive heart failure. New England J Med. 1991;325(5):293-302. doi:10.1056/ NEJM199108013250501

13. Colucci WS, Elkayam U, Horton DP, et al. Intravenous nesiritide, a natriuretic peptide, in the treatment of decompensated congestive heart failure. $N$ Engl $J$ Med. 2000;343(4):246-253. doi:10.1056/ NEJM200007273430403

14. O'Connor CM, Starling RC, Hernandez AF, et al. Effect of nesiritide in patients with acute decompensated heart failure. $N$ Engl $J$ Med. 2011;365(1):32-43. doi:10.1056/NEJMoa1100171

15. Yancy CW, Krum H, Massie BM, et al. Safety and efficacy of outpatient nesiritide in patients with advanced heart failure: results of the second follow-up serial infusions of Nesiritide (FUSION II) trial. Circ Heart Fail. 2008;1(1):9-16. doi:10.1161/CIRCHEARTF AILURE.108.767483

16. Chen HH, Anstrom KJ, Givertz MM, et al. Low-dose dopamine or low-dose nesiritide in acute heart failure with renal dysfunction: the ROSE acute heart failure randomized trial. JAMA. 2013;310 (23):2533-2543. doi:10.1001/jama.2013.282190

17. Newby DE, McDonagh T, Currie PF, et al. Candoxatril improves exercise capacity in patients with chronic heart failure receiving angiotensin converting enzyme inhibition. Eur Heart J. 1998;19 (12):1808-1813. doi:10.1053/euhj.1998.1201
18. Bonsu KO, Owusu IK, Buabeng KO, Reidpath DD, Kadirvelu A. Review of novel therapeutic targets for improving heart failure treatment based on experimental and clinical studies. Ther Clin Risk Manag. 2016;12:887-906. doi:10.2147/TCRM.S106065

19. Packer M, Califf RM, Konstam MA, et al. Comparison of omapatrilat and enalapril in patients with chronic heart failure: the omapatrilat versus enalapril randomized trial of utility in reducing events (OVERTURE). Circulation. 2002;106(8):920-926. doi:10.1161/01. CIR.0000029801.86489.50

20. Hubers SA, Brown NJ. Combined angiotensin receptor antagonism and neprilysin inhibition. Circulation. 2016;133(11):1115-1124. doi:10.1161/CIRCULATIONAHA.115.018622

21. Black HR, Bailey J, Zappe D, Samuel R. Valsartan: more than a decade of experience. Drugs. 2009;69(17):2393-2414. doi:10.2165/11319460000000000-00000

22. Desai AS, McMurray JJ, Packer M, et al. Effect of the angiotensin-receptor-neprilysin inhibitor LCZ696 compared with enalapril on mode of death in heart failure patients. Eur Heart $J$. 2015;36(30):1990-1997. doi:10.1093/eurheartj/ehv186

23. Packer M, McMurray JJ, Desai AS, et al. Angiotensin receptor neprilysin inhibition compared with enalapril on the risk of clinical progression in surviving patients with heart failure. Circulation. 2015;131(1):54-61. doi:10.1161/CIRCULATIO NAHA.114.013748

24. Yancy CW, Jessup M, Bozkurt B, et al. 2016 ACC/AHA/HFSA focused update on new pharmacological therapy for heart failure: an update of the 2013 ACCF/AHA guideline for the management of heart failure: a report of the American College of Cardiology/ American Heart Association task force on clinical practice guidelines and the Heart Failure Society of America. J Am Coll Cardiol. 2016;68(13):1476-1488. doi:10.1016/j.jacc.2016.05.011

25. Sokos GG, Raina A. Understanding the early mortality benefit observed in the PARADIGM-HF trial: considerations for the management of heart failure with sacubitril/valsartan. Vasc Health Risk Manag. 2020;16:41. doi:10.2147/VHRM.S197291

26. Simpson J, Jhund P, Rouleau J, et al. Effect of sacubitril/valsartan compared with enalapril, according to etiology in PARADIGM-HF. J Am Coll Cardiol. 2017;69((11 Supplement)):919. doi:10.1016/ S0735-1097(17)34308-5

27. Kristensen SL, Martinez F, Jhund PS, et al. Geographic variations in the PARADIGM-HF heart failure trial. Eur Heart J. 2016;37 (41):3167-3174. doi:10.1093/eurheartj/ehw226

28. Januzzi JL, Butler J, Fombu E, et al. Rationale and methods of the prospective study of biomarkers, symptom improvement, and ventricular remodeling during sacubitril/valsartan therapy for heart failure (PROVE-HF). Am Heart J. 2018;1(199):130-136. doi:10.1016/j. ahj.2017.12.021

29. Desai AS, Solomon SD, Shah AM, et al. Effect of sacubitril-valsartan vs enalapril on aortic stiffness in patients with heart failure and reduced ejection fraction: a randomized clinical trial. JAMA. 2019;322(11):1077-1084. doi:10.1001/jama.2019.12843

30. Senni M, McMurray JJV, Wachter R, et al. Initiating sacubitril/ valsartan (LCZ696) in heart failure: results of TITRATION, a double-blind, randomized comparison of two uptitration regimens. Eur J Heart Fail. 2016;18(9):1193-1202. doi:10.1002/ ejhf.548

31. Wachter R, Senni M, Belohlavek J, et al. Initiation of sacubitril/ valsartan in haemodynamically stabilised heart failure patients in hospital or early after discharge: primary results of the randomised TRANSITION study. Eur J Heart Fail. 2019;21(8):998-1007. doi:10.1002/ejhf. 1498

32. Senni M, Wachter R, Witte KK, et al. Initiation of sacubitril/valsartan shortly after hospitalisation for acutely decompensated heart failure in patients with newly diagnosed (de novo) heart failure: a subgroup analysis of the TRANSITION study. Eur J Heart Fail. 2020;22 (2):303-312. doi:10.1002/ejhf.1670 
33. Velazquez EJ, Morrow DA, DeVore AD, et al. Angiotensin-neprilysin inhibition in acute decompensated heart failure. $N \mathrm{Engl} \mathrm{J} \mathrm{Med.}$ 2019;380(6):539-548. doi:10.1056/NEJMoa1812851

34. Ambrosy AP, Mentz RJ, Fiuzat M, et al. The role of angiotensin receptor-neprilysin inhibitors in cardiovascular disease-existing evidence, knowledge gaps, and future directions. Eur J Heart Fail. 2018;20(6):963-972. doi:10.1002/ejhf.1159

35. Martens P, Verluyten L, Van de Broek H, et al. Determinants of maximal dose titration of sacubitril/valsartan in clinical practice. Acta Cardiol. 2019;6:1.

36. Haynes R, Judge PK, Staplin N, et al. Effects of sacubitril/valsartan versus irbesartan in patients with chronic kidney disease: A randomized double-blind trial. Circulation. 2018;138 (15):1505-1514. doi:10.1161/CIRCULATIONAHA.118.034818

37. Seferovic JP, Claggett B, Seidelmann SB, et al. Effect of sacubitril/ valsartan versus enalapril on glycaemic control in patients with heart failure and diabetes: a post-hoc analysis from the PARADIGM-HF trial. Lancet Diab Endocrinol. 2017;5(5):333-340. doi:10.1016/ S2213-8587(17)30087-6

38. Solomon SD, Zile M, Pieske B, et al. The angiotensin receptor neprilysin inhibitor LCZ696 in heart failure with preserved ejection fraction: a Phase 2 double-blind randomised controlled trial. Lancet. 2012;380(9851):1387-1395. doi:10.1016/S0140-6736(12)61227-6

39. Solomon SD, McMurray JJ, Anand IS, et al. Angiotensin-neprilysin inhibition in heart failure with preserved ejection fraction. New England $J$ Med. 2019;381(17):1609-1620. doi:10.1056/NEJ Moa1908655

40. Solomon S, of JM-TNEJ, 2020 undefined. Angiotensin-Neprilysin inhibition in heart failure with preserved ejection fraction. reply. europepmc.org. Available from: https://europepmc.org/article/med/ 32187481. Accessed June 21, 2020

41. JJV M, Jackson AM, Lam CSP, et al. Effects of sacubitril-valsartan versus valsartan in women compared with men with heart failure and preserved ejection fraction: insights from PARAGON-HF. Circulation. 2020;141(5):338-351. doi:10.1161/CIRCULATIO NAHA.119.044491
42. A randomized, double-blind controlled study comparing lcz696 to medical therapy for comorbidities in hfpef patients full text view clinicaltrials.gov. Available from: https://clinicaltrials.gov/ct2/show/ NCT03066804. Accessed June 21, 2020.

43. Study of efficacy and safety of LCZ696 in Japanese patients with chronic heart failure and reduced ejection fraction. full text view clinicaltrials.gov. Available from: https://clinicaltrials.gov/ct2/show/ NCT02468232. Accessed June 21, 2020.

44. Prospective ARNI vs ACE inhibitor trial to determine superiority in reducing heart failure events after MI full text view clinicaltrials.gov. Available from: https://clinicaltrials.gov/ct2/show/NCT02924727. Accessed June 21, 2020.

45. The effects of sacubitril/valsartan compared to valsartan on LV remodelling in asymptomatic LV systolic dysfunction after MI full text view clinicaltrials.gov. Available from: https://clinicaltrials.gov/ ct2/show/NCT03552575. Accessed June 21, 2020.

46. Differential vascular and endocrine effects of valsartan/sacubitril in heart failure with reduced ejection fraction full text view clinicaltrials.gov. Available from: https://clinicaltrials.gov/ct2/show/ NCT03168568. Accessed June 21, 2020.

47. Safety and efficacy of ARNI after LVAD implanT (SEAL-IT) study full text view clinicalTrials.gov. Available from: https://clinicaltrials. gov/ct2/show/NCT04191681. Accessed June 21, 2020

48. Sacubitril-valsartan and heart failure patients: the entresto-sas study full text view clinicaltrials.gov. Available from: https://clinicaltrials. gov/ct2/show/NCT02916160. Accessed June 21, 2020

49. Perlman A, Hirsh Raccah B, Matok I, Muszkat M. Cognition and dementia-related adverse effects with sacubitril-valsartan: analysis of the FDA adverse event report system database. J Card Fail. 2018;24 (8):533-536. doi:10.1016/j.cardfail.2018.04.010
Therapeutics and Clinical Risk Management

\section{Publish your work in this journal}

Therapeutics and Clinical Risk Management is an international, peerreviewed journal of clinical therapeutics and risk management, focusing on concise rapid reporting of clinical studies in all therapeutic areas, outcomes, safety, and programs for the effective, safe, and sustained use of medicines. This journal is indexed on PubMed Central, CAS,

\section{Dovepress}

EMBase, Scopus and the Elsevier Bibliographic databases. The manuscript management system is completely online and includes a very quick and fair peer-review system, which is all easy to use. Visit http://www.dovepress.com/testimonials.php to read real quotes from published authors. 